Pacific Journal of Mathematics

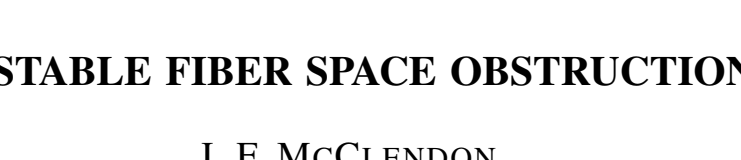




\section{ON STABLE FIBER SPACE OBSTRUCTIONS}

\section{J. F. MCCLENDoN}

\section{It will be proved that every stable fiber space obstruction} is a coset of the image of some stable twisted cohomology operation.

1. Statement of the theorem. Consider the following tower of $K$-principal fibrations over a path connected space $B$.

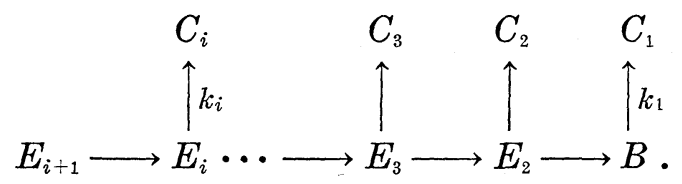

Each $C_{i}$ is a product, over $K=K(\pi, 1)$, of $L(G, n)^{\prime} s$. $\pi=\pi_{1}(B)$ and $L(G, n)=L_{\phi}(G, n)$ where $\phi: \pi \rightarrow$ aut $G$. (" $L_{\phi}(G, n)$ " and " $K$-principal fibration" are discussed in $\S 2$. They generalize " $K(G, n)$ " and "principal fibration".) Suppose $X$ is a $C W$ space and $f: X \rightarrow B$ is a given map. Define

$$
O_{i}(f)=\left\{\hat{f}^{*} k_{i} \mid \hat{f}: X \rightarrow E_{i} \text { is a lifting of } f\right\} \subset\left[X, C_{i}\right]_{K} \text {. }
$$

Then $O_{1}(f), O_{2}(f), \cdots, O_{i}(f)$, are the successive obstructions to lifting $f$ to $E_{i+1}\left(f\right.$ lifts to $E_{i+1}$ if and only if $\left.0 \in O_{j}(f), 1 \leqq j \leqq i\right)$. A more detailed description of local coefficient obstruction theory is given in [4]. The purpose of the present paper is to prove the following theorem.

TheOREM. Assume that there is an $N$ such that the $C_{j}$ 's are products of $L(G, n)$ 's and $N+1 \leqq n<2 N$. Then there is a stable $B$-operation $\Phi=\Phi_{x, f}:\left[X, \Omega C_{1}\right]_{K} \rightarrow\left[X, C_{i}\right]_{K}$ (an additive relation) such that $O_{i}(f)$ is a coset of the subgroup Image $\Phi \subset\left[X, C_{i}\right]_{K}$.

It will be seen from the proof that $O_{i}(f)$ is a coset under more general circumstances. The hypotheses stated here cover the case of a modified Postnikov system for a fibration $F \rightarrow E \rightarrow B$ (not necessarily orientable) when $\operatorname{dim} X \leqq 2 C, C=$ connectivity of $F$.

For simplicity, all spaces are assumed to be path connected, pointed and to have the homotopy type of $C W$ spaces. All maps are assumed pointed.

The result here seems to be new even for orientable systems. Results similar to this have been obtained, independently and earlier, by Mahowald [2] for orientable sphere bundles and Meyer [5] for 
orientable fibrations. However, neither shows that the obstruction is actually a coset.

2. Some definitions. First, recall from [Gitler 1] and [Siegel 7] the definition of $L_{\phi}(G, n)$. Let $\phi ; \pi \rightarrow$ aut $G$ be a homomorphism of a group $\pi$ into the automorphism group of an abelian group $G$. Then there is an associated map $\bar{\phi}$ from $\pi$ into the group of base point preserving homeomorphisms of $K(G, n)(K(G, n)$ is a pointed $C W$ space which is an Eilenberg-MacLane space of type $(G, n))$. The following diagram is commutative

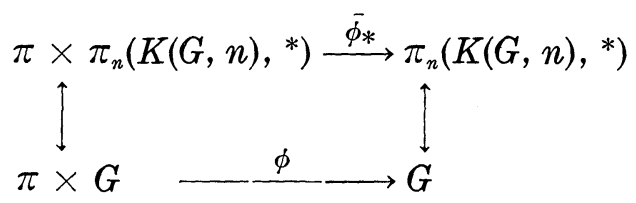

where $\bar{\phi}_{*}(x, y)$ means $\bar{\phi}(x)_{*}(y)$. Let $\pi \rightarrow \hat{L} \rightarrow K$ be the universal cover of $K=K(\pi, 1)$. It is a universal principal $\pi$-bundle. Let $L_{\phi}(G, n) \rightarrow$ $K(\pi, 1)$ be the associated bundle with fiber $K(G, n)$. It has a natural section. $(L, K)$ can be assumed to have the homotopy type of a $C W$ pair. Let $\Gamma$ be a local coefficient system on a space $X$ classified by $x: X \rightarrow K=K(\pi, 1)$. Then $H^{n}(X: \Gamma) \leftrightarrow[X, L]_{K}$ where the latter is the set of homotopy classes of maps over $K$ (see [Steenrod, 8] or [Olum, 6]).

Now suppose, in general, that $D \stackrel{\check{\omega}}{\longrightarrow} W \stackrel{\hat{\omega}}{\longrightarrow} D$ and $\hat{\omega} \check{\omega}=$ identity. Define

$$
\begin{aligned}
& \bar{P} W=\left\{k \in W^{I} \mid \hat{\omega} k(t)=\hat{\omega} k\left(t^{\prime}\right), t, t^{\prime} \in I, k(0)=\hat{\omega} \check{\omega}(0)\right\} \\
& \bar{\Omega} W=\{k \in \bar{P} W \mid k(0)=k(1)\} .
\end{aligned}
$$

If $W \rightarrow D$ is a fibration then $\bar{P} W \rightarrow W(k \rightarrow k(1))$ is a fibration with fiber $\Omega W$ (the ordinary loop space). Call it and any fibration induced from it a $D$-principal fibration. The following diagram is a pullback.

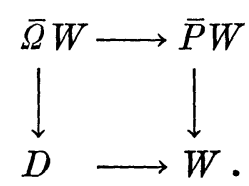

Now apply these definitions to $K(\pi, n) \rightarrow L_{\phi}(G, n) \rightarrow K(\pi, 1)$. $\bar{\Omega} L$ is $L_{\phi}(G, n-1)$. $f: X \rightarrow L$ lifts to $\bar{P} L$ if and only if $f$ is zero as an element of $H^{n}(X ; \Gamma)$ if and only if $f$ factors up to homotopy through $K \rightarrow L$.

Next, define $L_{D}(G, n)=L_{D, \phi, \alpha}(G, n)$ as follows. Suppose $\alpha: \pi_{1}(D) \rightarrow \pi$ is a given homomorphism corresponding to a map $D \rightarrow K(\pi, 1)$ also 
denoted by $\alpha$. Define $L_{D}(G, n)$ as the pullback of $\alpha$ and $L_{\phi}(G, n) \rightarrow$ $K(\pi, 1)$. If the classifying map for a local coefficient system $\Gamma$ on $X$ can be factored through $\alpha$ then $H^{n}(X, \Gamma) \leftrightarrow\left[X, L_{D}(G, n)\right]_{D}$. Suppose $\pi_{1}(D)=\pi, \alpha=$ the classifying map for $\Gamma$, and $\phi$ determines $\Gamma$. Then write $L_{T}(G, n)$ for $L_{D, \phi, \alpha}(G, n)$.

3. Twisted operations with local coefficients. Let $\Gamma$ and $\Gamma^{\prime}$ be local coefficient systems on a space $D$ determined by $\phi: \pi \rightarrow$ aut $G$ and $\Psi: \pi \rightarrow$ aut $H$ respectively, where $\pi=\pi_{1}(D)$. Let $x: X \rightarrow D$ be given. A primary operation $\Phi_{X, x}: H^{n}\left(X ; x^{*} \Gamma\right) \rightarrow H^{k}\left(X ; x^{*} \Gamma^{\prime}\right)$ is represented by a map from $\left(L_{T}(G, n), D\right)$ to $\left(L_{\phi}(H, k), K(\pi, 1)\right)$ which extends the classifying map for $\Gamma^{\prime}$. This map determines a unique map from $L_{\Gamma}(G, n)$ to $L_{\Gamma^{\prime}}(H, k)$ which is a map in $T D=$ the category of spaces under and over $D$ (see [3]).

Higher order operations can be defined by towers of $K(\pi, 1)$-principal fibrations such as the following one:

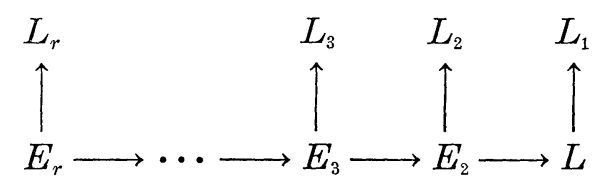

where $L=L_{\Gamma}(G, n), L_{r}=L_{\Psi}(H, k)$, and $L_{j}=L_{\phi_{j}}\left(G_{j}, n_{j}\right), \phi_{j}: \pi \rightarrow \operatorname{aut} G_{j}$, $1 \leqq j \leqq r-1$, and $E_{0} \rightarrow L_{j}$ sends $D$ to $K(\pi, 1)$. More specifically, $\Phi$ is defined by the following commutative diagram:

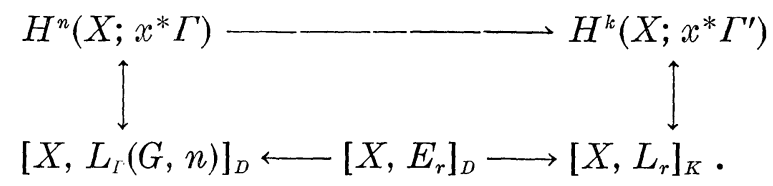

The $L$ 's may be replaced by products over $K(\pi, 1)$ to give operations in several variables.

Suppose in the stable case that $f: D \rightarrow B$ is given and that all coefficient systems for the above tower are obtained by pullback from systems over $B$. Assume also that $f^{*}: H^{*}(B) \rightarrow H^{*}(D)$ is isomorphic for $i<N$ and for all coefficient systems involved. Then there is a $B$-operation $\Psi$ such that if $X \stackrel{x}{\longrightarrow} D \stackrel{f}{\longrightarrow} B$ the operations $\Phi_{X, x}$ and $\Psi_{X, f x}$ are the same. This is proved by constructing a tower over $B$ whose pullback is the given tower which defines the $D$-operation $\Phi$.

The following lemma accounts for the "additive" in the main theorem. 
Lemma. If $N+1 \leqq n_{j}<2 N$ for all $n_{j}$ then the operations defined above are additive relations.

The proof will be given for secondary operations of one variable. The argument for higher order operations of several variables is quite similar (see [3] for the constant coefficient case).

First, we recall some facts from [3]. Let $D$ be any space. Top $D$ is defined to be the category whose objects are triples $(W, \breve{w}, \widehat{w})$ with $\breve{w}: D \rightarrow W, \widehat{w}: W \rightarrow D$, and $\widehat{w} \breve{w}=$ identity. The morphisms are continuous functions $f: W \rightarrow W^{\prime}$ such that $\widehat{w} f=\check{w}^{\prime}$ and $f \check{w}=\breve{w}^{\prime}$. Homotopy is defined in the natural way. The path and loop functors are defined as in $\S 2$. There are cone and suspension functors also. All of the basic properties of Top (pt) remain valid for Top $D$ (Top $(p t)$ is just the category of pointed spaces and maps). If $W, W^{\prime} \in \operatorname{Top} D$, let $\left\langle W, W^{\prime}\right\rangle$ be the set of homotopy classes of maps from $W$ to $W^{\prime}$. If $W^{\prime}$ is a [double] loop space in Top $D$ then $\left\langle W, W^{\prime}\right\rangle$ is a natural [Abelian] group. If $f: W \rightarrow W^{\prime}$ is a loop map then $f_{*}$ is a homomorphism.

Proof of the lemma for primary operations. Let $\alpha$ be a primary operation. By definition $\alpha$ is represented by a map in Top $D$ (also denoted by $\alpha$ ) $\alpha: L_{\Gamma}(G, n) \rightarrow L_{\Gamma^{\prime}}\left(H, n^{\prime}\right)$. If $n^{\prime}<2 n$ (as in the present theorem) then it can be shown that $\alpha$ is a loop map in Top $D$ so the operation is a homomorphism.

The map $\bar{P} W \rightarrow W$ defined by $l \rightarrow l(1)$ is a fibration in Top $D$. Any fibration induced from it by a map to $W$ (classifying map) is called a principal fibration. If the classifying map is a loop map then the total space of the principal fibration is a loop space.

Consider the following two diagrams

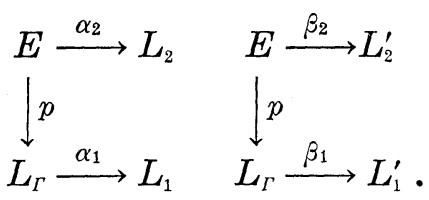

$L_{\Gamma}=L_{T}(G, n), L_{i}=L_{\phi(i)}\left(G_{i}, n_{i}\right), L_{i}^{\prime}=L_{\Gamma(i)}\left(G_{i}, n_{i}\right)=D \times{ }_{K} L_{i} \cdot \quad \Gamma, \Gamma_{i}$ are three given local coefficient systems on $D$ classified by $\phi: \pi \rightarrow$ Aut $G$, $\phi_{i}: \pi \rightarrow$ Aut $G_{i}$. $\quad \beta_{i}=\left(\mu, \alpha_{i}\right)$ where $u$ is the map to $D$ obtained from $E \rightarrow L_{T} \rightarrow D$. The important point is that $p: E \rightarrow L_{\Gamma}$ is the principal fibration induced by $\beta_{1}$ in Top $D$. This can be proved directly from the definitions. 
Proof of the lemma for secondary operations. Consider the following commutative diagram.

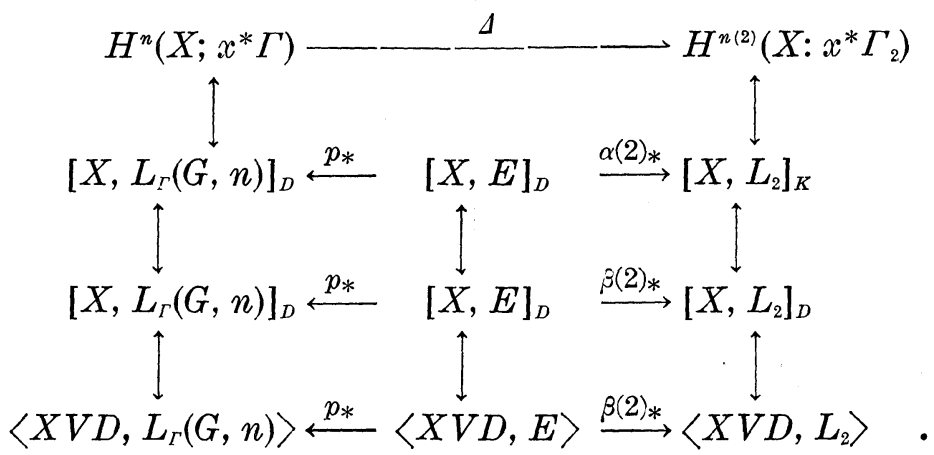

$\Delta$ is the secondary operation defined by line 2 . The diagram shows that $\Delta=\beta(2)_{*} p_{*}^{-1}$ via line 4 . Line 4 is obtained from Top $D . \quad p: E \rightarrow$ $L_{\Gamma}$ is a loop map and $L_{2}$ is a loop space in Top $D$. It can be shown that the dimension hypothesis of the lemma implies that $\beta(2)$ is a loop map in Top $D$. Hence $p_{*}$ and $\beta(2)_{*}$ are both homomorphisms and $\Delta$ is an additive relation.

If $D=K(\pi, 1)=K$ the operations discussed above are closely related to those defined by Siegel in [7]. The difference is as follows. Let local coefficient systems $\Gamma$ and $\Gamma^{\prime}$ on $K(\pi, 1)$ be represented by

$$
L_{\phi}(G, n) \underset{\check{l}}{\stackrel{\hat{\imath}}{\rightleftarrows}} K(\pi, 1) \quad L_{\psi}(H, k) \underset{\check{m}}{\stackrel{\hat{m}}{\rightleftarrows}} K(\pi, 1) .
$$

Then in the present paper a primary operation is represented by a map $f: L_{\phi}(G, n) \rightarrow L_{\psi}(H, k)$ which is a $K$-map, i.e., $\hat{m} f=\hat{l}$ and $f \grave{l}=\check{m}$. In Siegel's paper [7], a primary operation may be represented by a map which is merely a map over $K$, i.e., $\hat{m} f=\hat{l}$. The narrower definition of the present paper excludes the "characteristic operations" of [1, 7]. The advantage of the present approach is that all of the primary operations in the stable range are homomorphisms and the higher operations are additive relations. Presumably, the broader definition of [7] is more suitable for non-stable obstruction theory.

4. Proof of the theorem. Consider the following diagram where the left hand column is obtained from the right hand column by pullback. Let $s_{j}: E_{i} \rightarrow E_{i, j}$ be the natural section for the projection $p_{j}: E_{i, j} \rightarrow E_{i}$. Let $k_{j}^{\prime}$ be the composition

$$
E_{i, j} \longrightarrow E_{j} \stackrel{k_{i}}{\longrightarrow} C_{j}
$$




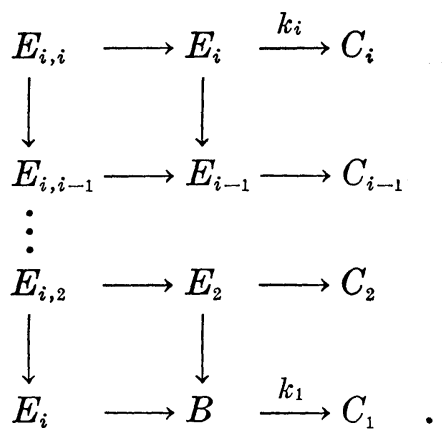

Claim. (1) $j \leqq i-1$ implies $k_{j}^{\prime} s_{j} \sim 0$ (over $K$ ).

(2) $k_{i}^{\prime}=\bar{k}_{i}+u, k_{i} p_{i}=\bar{k}_{i}, \bar{k}_{i} s_{i}=k_{i}$, and $u s_{i} \sim 0$ (over $K$ ).

Proof of claim. (1) is clear since $k_{j}^{\prime} s_{j}=E_{i} \rightarrow E_{j} \rightarrow C_{j} \sim 0$ (over $K)$. Write $\bar{E}$ for $E_{i, i}$ so

$$
\left(\bar{E}, E_{i}\right) \underset{p=p_{i}}{\stackrel{\stackrel{s=s_{i}}{D^{\prime}}}{\supset E_{i}}} \text { ps }=1 .
$$

Hence

$$
0 \rightarrow H^{*}\left(\bar{E}, E_{i}\right) \stackrel{g^{*}}{\longrightarrow} H^{*}(\bar{E}) \underset{p^{*}}{\stackrel{s^{*}}{\rightleftarrows}} H^{*}\left(E_{i}\right) \rightarrow 0
$$

where $H^{*}$ is $\left[, C_{i}\right]_{K} . \quad k_{i}^{\prime} s_{i}=k_{i}$, that is, $s^{*} k_{i}^{\prime}=k_{i}$. Hence $k_{i}^{\prime}=\bar{k}_{i}+u$ where $\bar{k}_{i}=p^{*} k_{i}$ and $u=g^{*} u^{\prime}$ and $u$ and $\bar{k}_{i}$ are unique. Hence $\bar{k}_{i} s_{i}=$ $k_{i} p s=k_{i}$. Also $k_{i}=k_{i}^{\prime}=\bar{k}_{i} s_{i}+u s_{i}=k_{i}+u s_{i}$ implying $u s_{i}=0$. This proves the claim.

Consider the following diagram:

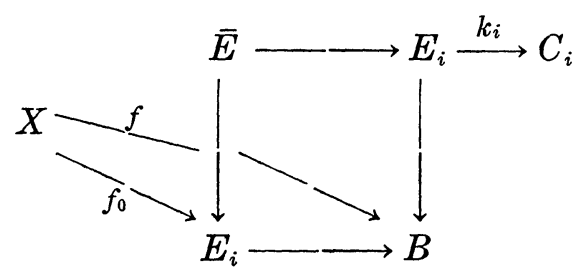

where $f_{0}$ is a fixed lifting of $f$ to $E_{i}$. Let $k=k_{i}, k^{\prime}=k_{i}^{\prime}$, and $E=E_{i}$. Then $O_{i}(f)=\left\{\hat{f}^{*} k \mid \hat{f}: X \rightarrow E\right.$ lifts $\left.f\right\}=\left\{\bar{f}^{*} k^{\prime} \mid \bar{f}: X \rightarrow \bar{E}\right.$ lifts $\left.f_{0}\right\}$ (since the liftings of $f$ and $f_{0}$ are in one-to-one correspondence)

$$
\begin{aligned}
& =\left\{\bar{f}^{*} \bar{k}+\bar{f}^{*} u\right\}=\bar{f}^{*} \bar{k}+\left\{\bar{f}^{*} u \mid \bar{f} \text { lifts } f_{0}\right\} \\
& =\bar{f}^{*} \bar{k}+\left\{\bar{f}^{*} u \mid \bar{f} \text { lifts } f_{0}^{\prime}: X \rightarrow E_{i, 2} \text { and } f_{0}^{\prime} \text { lifts } f_{\mathrm{c}}\right\} \\
& =\bar{f}^{*} \bar{k}+\Phi_{X, f_{0}}\left[X, \bar{\Omega} C_{1}\right]_{K} .
\end{aligned}
$$

Use: $E_{i, 2}=E_{i} \times{ }_{K} \bar{P} C_{1},\left[X, E_{i, 2}\right]_{\Sigma_{i}}=\left[X, \bar{P} C_{1}\right]_{K}=\left[X, \bar{\Omega} C_{1}\right] . \quad \Phi$ is the $E_{i^{-}}$ 
operation defined by $E_{i, 2} \leftarrow \bar{E} \rightarrow C_{i}$. If $C_{1}$ is the product of $L(G, n)^{\prime} s$ over $K$ then $E_{i, 2}$ is a corresponding product over $E_{i}$ of $L_{E_{i}}(G, n-1)^{\prime} s$. This shows that $O_{i}(f)$ is a coset of the image of the stable $E$ operation $\Phi$ defined by $E_{i, 2} \leftarrow \bar{E} \rightarrow C_{i}$. The hypotheses of the theorem and the remarks in the third paragraph of $\S 3$ guarantee that there is a stable $B$-operation $\Psi$ with the same domain and image. This completes the proof.

\section{REFERENCES}

1. S. Gitler, Cohomology operations with local coefficients, Amer. J. Math. 85 (1963), 156-188.

2. M. Mahowald, The third and fourth obstructions in sphere bundles (1962?), unpublished.

3. J. F. McClendon, Higher order twisted cohomology operations, Invent. Math. 7 (1969), 183-214.

4. Obstruction theory in fiber spaces (Submitted for publication)

5. J.P. Meyer, The indeterminacy of certain cohomology operations (1968?), unpublished.

6. P. Olum, On mapping into spaces in which certain homotopy groups vanish, Ann. of Math. 57 (1953), 561-573.

7. J. Siegel, Higher order cohomology operations in local coefficient theory, Amer. J. Math. 89 (1967), 909-931.

8. N. Steenrod, The Topology of Fiber Bundles, Princeton University Press, Princeton, 1951.

Received November 5, 1969, and in revised form June 8, 1970. This research was partly supported by a grant from the University of Kansas.

UNIVERSITY OF KANSAS 



\section{PACIFIC JOURNAL OF MATHEMATICS}

\section{EDITORS}

H. SAMELson

Stanford University

Stanford, California 94305

R. R. PHelPS

University of Washington

Seattle, Washington 98105
J. Dugundu

Department of Mathematics

University of Southern Californle

Los Angeles, California 9.0007

RICHARD ARENS

University of California

Los Angeles, California 9.0024

\section{ASSOCIATE EDITORS}
E. F. BECKENBACH
B. H. NeumanN
F. WOLE
K. Yoshida

\section{SUPPORTING INSTITUTIONS}

UNIVERSITY OF BRITISH COLUMBIA

CALIFORNIA INSTITUTE OF TECHNOLOGY

UNIVERSITY OF CALIFORNIA

MONTANA STATE UNIVERSITY

UNIVERSITY OF NEVADA

NEW MEXICO STATE UNIVERSITY

OREGON STATE UNIVERSITY

UNIVERSITY OF OREGON

OSAKA UNIVERSITY

UNIVERSITY OF SOUTHERN CALIFORNIA

\author{
STANFORD UNIVERSITY \\ UNIVERSITY OF TOKYO \\ UNIVERSITY OF UTAH \\ WASHINGTON STATE UNIVERSITY \\ UNIVERSITY OF WASHINGTON \\ AMERICAN MATHEMATICAL SOCIETY \\ CHEVRON RESEARCH CORPORATION \\ NAVAL WEAPONS CENTER
}

The Supporting Institutions listed above contribute to the cost of publication of this Journal, but they are not owners or publishers and have no responsibility for its content or policies.

Mathematical papers intended for publication in the Pacific Journal of Mathematics should be in typed form or offset-reproduced, (not dittoed), double spaced with large margins. Underline Greek letters in red, German in green, and script in blue. The first paragraph or two must be capable of being used separately as a synopsis of the entire paper. The editorial "we" must not be used in the synopsis, and items of the bibliography should not be cited there unless absolutely necessary, in which case they must be identified by author and Journal, rather than by item number. Manuscripts, in duplicate if possible, may be sent to any one of the four editors. Please classify according to the scheme of Math. Rev. Index to Vol. 39. All other communications to the editors should be addressed to the managing editor, Richard Arens, University of California, Los Angeles, California, 90024.

50 reprints are provided free for each article; additional copies may be obtained at cost in multiples of 50 .

The Pacific Journal of Mathematics is published monthly. Effective with Volume 16 the price per volume (3 numbers) is $\$ 8.00$; single issues, $\$ 3.00$. Special price for current issues to individual faculty members of supporting institutions and to individual members of the American Mathematical Society: $\$ 4.00$ per volume; single issues $\$ 1.50$. Back numbers are available.

Subscriptions, orders for back numbers, and changes of address should be sent to Pacific Journal of Mathematics, 103 Highland Boulevard, Berkeley, California, 94708.

PUBLISHED BY PACIFIC JOURNAL OF MATHEMATICS, A NON-PROFIT CORPORATION

Printed at Kokusai Bunken Insatsusha (International Academic Printing Co., Ltd.), 7-17, Fujimi 2-chome, Chiyoda-ku, Tokyo, Japan. 


\section{Pacific Journal of Mathematics}

\section{Vol. 36, No. 2 December, 1971}

George E. Andrews, On a partition problem of H. L. Alder ............ 279

Thomas Craig Brown, An interesting combinatorial method in the theory of locally finite semigroups .......................... 285

Yuen-Kwok Chan, A constructive proof of Sard's theorem ............. 291

Charles Vernon Coffman, Spectral theory of monotone Hammerstein

operators...................................... 303

Edward Dewey Davis, Regular sequences and minimal bases .......... 323

Israel (Yitzchak) Nathan Herstein and Lance W. Small, Regular elements in

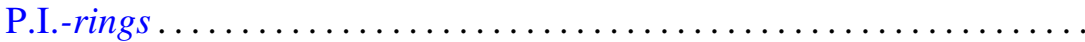

Marcel Herzog, Intersections of nilpotent Hall subgroups ..............

W. N. Hudson, Volterra transformations of the Wiener measure on the space

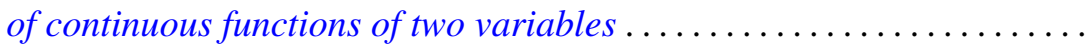

J. H. V. Hunt, An n-arc theorem for Peano spaces ................ 351

Arnold Joseph Insel, A decomposition theorem for topological group

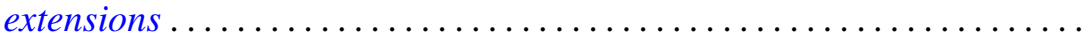

Caulton Lee Irwin, Inverting operators for singular boundary value

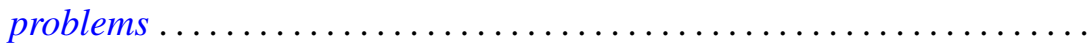

Abraham A. Klein, Matrix rings of finite degree of nilpotency ............ 387

Wei-Eihn Kuan, On the hyperplane section through a rational point of an algebraic variety...

John Hathway Lindsey, II, On a six-dimensional projective representation of $\mathrm{PSU}_{4}(3)$

Jorge Martinez, Approximation by archimedean lattice cones ...

J. F. McClendon, On stable fiber space obstructions .........

Mitsuru Nakai and Leo Sario, Behavior of Green lines at the Kuramochi boundary of a Riemann surface ....................

Donald Steven Passman, Linear identities in group rings. I. .

Donald Steven Passman, Linear identities in group rings. II ...

David S. Promislow, The Kakutani theorem for tensor products of

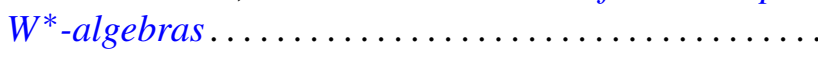

Richard Lewis Roth, On the conjugating representation of a finite group

Bert Alan Taylor, On weighted polynomial approximation of entire functions...

William Charles Waterhouse, Divisor classes in pseudo Galois

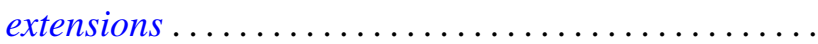

Chi Song Wong, Subadditive functions ...

Ta-Sun $\mathrm{Wu}$, A note on the minimality of certain bitransformation groups 